\title{
LA EDUCACIÓN EN LA SEGUNDA REPUBLICA LIBERAL (1930-1946) Apuntes para una historiografía
}

\section{Martha Cecilia Herrera*}

La investigación sobre educación en Colombia cobra su mayor impulso hacia 1960 y décadas subsiguientes, cuando desde diferentes perspectivas estudiosos de las ciencias sociales abordaron la descripción y análisis de las diversas manifestaciones del fenómeno educativo. Los trabajos aparecen en primera instancia ligados a la demografía y a la planificación económica, quedando circunscritos o bien a los aspectos de cuantificación que precisaban el número de establecimientos, alumnos, maestros, etc., o a los elementos descriptivos de la legislación educativa. Ellos no establecieron relaciones significativas con los procesos históricos, con los hechos socio-económicos, con los mecanismos ideológicos y políticos, ni con la dinámica e inserción de la educación en la vida cotidiana.

A nivel de descripciones globales en el ámbito de lo que tradicionalmente se ha conocido como Historia de la Educación, se publicaron entre las décadas del 50 y 70 estudios como los de Hoenisberg ${ }^{53}$, Rodríguez Rojas ${ }^{54}$, Bohórquez Casallas ${ }^{55}$, Escobar Bernal $^{56}$, los cuales esbozaron las principales coordenadas de la educación en los diferentes períodos históricos. Posteriormente algunos investigadores profundizaron ciertos puntos o llevaron a cabo síntesis a partir de estos autores. En realidad puede afirmarse que el campo de la Historia de la Educación ha sido poco explorado y aunque existen esfuerzos valiosos, en gran parte se han limitado a la consulta de fuentes secundarías y a la mirada juridicista situada en el estrecho prisma de la legislación educativa, sin que los aportes superen los trabajos pioneros ${ }^{57}$.

En lo que se refiere a la educación en el siglo $X X$, la producción histórica se ha centrado con preferencia en su segunda mitad, en la que se presentaron complejos fenómenos de expansión demográfica al tiempo que un aumento significativo de la escolaridad que llevó a cambios en las estructuras educativas. Sobre las primeras décadas del presente siglo existen pocas investigaciones, si exceptuamos las referencias someras en obras de carácter general, no obstante, en este período se sentaron las bases de los procesos educativos que ocurrieron después de 1950.

Sobre las políticas educativas en el siglo $X X$ y la concepción que sobre ellas tuvieron los partidos tradicionales, se pueden identificar dos posiciones contrapuestas, una que juzga la mayoría de las acciones liberales como progresistas, o por el contrario, ópticas como la de Bohórquez que ubican las reformas implementadas en las administraciones conservadoras como las más positivas.

\footnotetext{
* Socióloga y Magíster en Historia de Colombia. Profesora de la Universidad Pedagógica Nacional. Área de Formación Pedagógica y Didáctica.

${ }^{53}$ HOENISBERG, Julio. Las fronteras de los partidos en Colombia. Historia y comentarios de la legislación escolar en la República desde 1821 hasta el 13 de Junio de 1953. Bogotá, 1953.

${ }^{54}$ RODRIGUEZ ROJAS, José María. "Historia de la educación en Colombia”. En: Rey, de la Universidad de Antioquia, Vol. 1-2, 1954-55.

${ }^{55}$ BOHORQUEZ CASAL LAS, Luis A. La evolución educativa en Colombia. Bogotá, 1956.

${ }^{56}$ ESCOBAR BERNAL, Alejandro y otros. La educación en la historia de Colombia. lcodes, Bogotá, 1964.

${ }^{57}$ En aras al tratamiento del tema central —Educación y República Liberal— no nos detendremos en el análisis sobre los textos anteriores, por el momento nos interesa dejar sentado que son estos los primeros acercamientos a la problemática.

La Reforma Constitucional de 1936. Fundación Newman, Bogotá, 1984.

Lo económico y lo social en la Reforma Constitucional del 36". En: Estado y economía. Cincuenta años de la Reforma del 36.

Contraloría General de la República, Bogotá, 1986. pp. 9-24.
} 
Por encima de estos dos enfoques se abre paso una corriente renovadora que sitúa sus análisis en un espectro más amplio. Es el caso por ejemplo, de la historiadora suiza Aline Helg, quien se atreve a cuestionar la interpretación usual sobre la radical diferencia entre políticas educativas liberales y conservadoras, afirmando que "de opuestas que eran en el siglo XIX... evolucionaron hacia la similitud mucho antes del Frente Nacional"

Helg esgrime como principal argumento de las discrepancias entre liberales y conservadores en la primera parte del siglo XX, no tanto las divergencias ideológicas profundas, como sí las rencillas ocasionadas por la apropiación burocrática de numerosos empleos, fruto de la ampliación de la acción estatal en los diversos órdenes de lo social, entre los que se contaba, el sistema educativo ${ }^{59}$. Para la década del 50 las divergencias entre las élites han sido limadas en aras a su unidad en un frente común, que busca oponer resistencia a la enconada lucha de clases que desbordaba los marcos institucionales.

Aunque la hipótesis de Helg sobre la falsa diferenciación entre políticas educativas propuestas por liberales y conservadores merece una mayor fundamentación, tiene el valor de alejarse de las fronteras de interpretación ya trajinadas, al sustraer el análisis de la concepción maniqueísta liberal, que ha utilizado la pareja reacción-progreso para colocar su actuar político y administrativo como el de avanzada; sin embargo también los conservadores han juzgado ser ellos quienes han protagonizado los cambios en materia educativa.

La polémica aún no ha concluido, es preciso ahondar sobre ella enriqueciéndola y sobre todo, es muy importante develar las expectativas que subyacen en las explicaciones sobre este período histórico. Es por ello que el historiador requiere someter a juicio permanente y análisis crítico los documentos con los que trabaja, tener en cuenta y sopesar tanto sus intereses como los de los actores sociales sobre los sucesos que observa y establecer un diálogo contínuo entre pasado y presente ${ }^{60}$.

Delineados estos aspectos generales, procederemos a tratar específicamente la producción histórica ceñida a la República Liberal 1930-1 946. Se presentarán las principales investigaciones, las diversas vías interpretativas sus aportes, vacíos e inconsistencias con el fin de hacer una primera aproximación al estado en que se encuentra este problema.

En su gran mayoría los trabajos no están ausentes de las simpatías partidistas a las que ya hicimos referencia. Así, dentro de la tendencia historio-gráfica que considera progresistas las políticas desarrolladas durante la República Liberal, y de manera especial, el primer mandato presidencial de Alfonso López Pumarejo, persiste un enfoque que se quedó en el recuento de la historia de jas ideas desligado de otros aspectos que puedan confrontarla en diversos terrenos ${ }^{61}$.

Al lado de la anterior tendencia existe otra que no se ha quedado en el nivel legislativo, en los discursos y programas políticos para el examen del período, entrando a sopesar las ideas desde otros ángulos cotejándolas con las realizaciones prácticas. Esta visión es

\footnotetext{
${ }^{58}$ HELG, Aline. "Liberales versus conservadores en la educación colombiana, un mito?". Facultad de Educación de la Universidad de Ginebra e Instituto Universitario de Estudios del Desarrollo. Mimeo, Bogotá, Septiembre 1984.

${ }^{59}$ La prensa del período expresa las contradicciones motivadas por el nombramiento de funcionarios estatales, pertenecientes a uno y otro partido. Este hecho es también reseñado por el sociólogo e historiador Daniel Pecaut.

${ }^{60}$ CARR, E.H. ¿Qué es la historia? Seix Barral, Barcelona, 1978. p. 40.

${ }^{61}$ El historiador Alvaro Tirado es sin duda una de las principales figuras de esta tendencia:

TIRADO MEJIA, Alvaro. Aspectos políticos de las reformas de Alfonso López Pumarejo (1934-1938). Procultura, Bogotá, 1981.
} 
más crítica frente a los alcances de las administraciones liberales y apoyada en estudios económicos, políticos y sociales cuestiona en buena parte, la retórica de las políticas gubernamentales y la interpretación apologética que comúnmente se les ha dado ${ }^{62}$.

Una tercera corriente de corte conservador indica el período liberal como nefasto para la economía y la sociedad en su conjunto, matiz que influirá en las explicaciones sobre la educación 63 .

Veamos ahora como se expresan estas posiciones en el ámbito educativo a través de investigadores como Ivon Lebot, Jaime Jaramillo Uribe, Fernán González, Molano y Vera, Aline Helg, Antonio García y Luis A. Bohórquez.

En su ensayo Ivon Lebot $(1972)^{64}$, hace un rápido recorrido por las políticas educativas del siglo XX, aportando elementos para la exégesis y adjuntando datos estadísticos que corroboran sus apreciaciones. No se detiene en el estudio riguroso de las fuentes primarias, busca más bien esclarecer los principales rasgos de la educación, eligiendo como hilo conductor la legislación basado en obras de carácter secundario como la de Bohórquez.

Habla de transformaciones educativas que germinaron entre las décadas del 20 al 40 , que encuentran en parte explicación en la existencia de una burguesía nacional que "abriéndose paso impugna las formas tradicionales de la estructura social y de la superestructura", pretendiendo dentro de una "perspectiva nacionalista" hacer de la educación un factor de "integración nacional ".

Este autor señala, empero, las limitaciones de las reformas y la inclinación de la política educativa a privilegiar la educación media y superior, en detrimento de la educación primaria y la alfabetización, que constituían paradójicamente el pilar de la bandera de "educación popular", enarbolada en los discursos de los dirigentes liberales de la época.

Jaime Jaramillo Uribe $(1980)^{65}$, aborda con una perspectiva histórica global todo el proceso de la educación desde la colonia hasta nuestros días. Su trabajo contiene interesantes apreciaciones que sirvieron de guía a otros estudios. Presenta un tratamiento desigual de la temática, pues si bien hay partes muy trabajadas y con apoyo documental serio, otras carecen de esfuerzos sistemáticos y se limitan a retomar lo que ya se ha dicho, en especial en lo que se refiere al siglo XX para el que se vale en gran medida en las obras de Bohórquez y Lebot.

Sobre los cambios educativos en la República Liberal, Jaramillo Uribe afirma que ellos son coherentes con el espíritu reformista de los liberales, el que ya tenía expresión desde el siglo XIX. Indica los avances en materia educativa, para concluir que a la postre "se intentó sólo una limitada reforma de carácter positivista en su contenido ideológico, sin

\footnotetext{
${ }^{62}$ MONCAYO y ROJAS. Luchas obreras y política laboral en Colombia. La Carreta, Bogotá, 1978.

PECAUT, Daniel. Política y Sindicalismo en Colombia. La Carreta, Bogotá, 1973.

VEGA, Renán. "Colombia en la época de la Segunda Guerra Mundial: Contraofensiva oligárquica y antirrevolución política". Texto de conferencia dictada en la Universidad lncca, Mayo de 1985.

"Liberales al poder". En: Historia de Colombia. Oveja Negra, Bogotá, 1986. Fascículo

${ }^{63}$ No conocemos obras recientes con una visión conservadora de la República Liberal, pues el libro de Martín Alonso Pinzón sobre la "Historia del Conservatismo", publicado en 1979 sólo alcanza a hacer breves referencias sobre el período, sin embargo en la época se editaron dos textos en los que quedó consignado este tipo de interpretación histórica de las realizaciones del partido liberal entre 1930 y 1946:

SALAMANCA, Guillermo. La República Liberal. Editorial Centro, Bogotá, 1937. 2v.

SALDARRIAGA BETANCUR, Juan Manuel. El Régimen del terror o 76 años en el infierno. Imprenta Departamental, Medellín, 1951.

${ }^{64}$ LEBOT, Ivon. Educación e Ideología en Colombia. La Carreta, Medellín, 1979.

${ }^{65}$ JARAMILLO URIBE, Jaime. "El proceso de la educación del virreinato a la época contemporánea". En: Manual de Historia de Colombia, Tomo III. Colcultura, Bogotá, 1980. pp. 243-339.
} 
alcanzar a resolver el problema básico del analfabetismo, ni a plantear, y menos a poner en práctica el ideal de la escuela pública elemental, obligatoria y única para todas las clases sociales como base de la unidad nacional”"66.

Por la gran cobertura de la síntesis de Jaramillo U. las hipótesis sobre la República Liberal no se tratan como hipótesis a demostrar. Presenta una marcada ausencia respecto a la polémica Iglesia-Estado sobre la orientación y control de la educación, ocurrida a raíz de la reforma educativa de 1936, omitiendo uno de los aspectos más relevantes del período.

La obra de Fernán González (1978) ${ }^{67}$, da cuenta de los principales acontecimientos del devenir educativo en el país y su relación con el Estado, incluyendo algunos apuntes sobre el sector privado. Elabora un resumen con carácter descriptivo basado en lo fundamental en bibliografía secundaria. Sobre la educación entre 1930 y 1946 dice siguiendo a Lebot- que "la crisis interna del sistema oligárquico y las repercusiones de la crisis mundial, acompañadas del surgimiento de un intento de burguesía nacional repercute en una nueva orientación educativa". Asevera que los mayores logros se dieron en el sector universitario. Reseña la polémica Iglesia-Estado referido al libro de Hoenisberg, sin recurrir a documentación primaria que enriquezca las apreciaciones de este autor liberal. Sus líneas sobre la República Liberal no son muy extensas, se limitan a los hechos más sobresalientes de la reforma educativa, sin contrastar las disposiciones legislativas con sus implementaciones prácticas.

Molano y Vera $(1982)^{68}$, retoman la política educativa a lo largo del siglo XX, sustentados en el uso de fuentes secundarias y en la consulta no muy exhaustiva de información de prensa. El enfoque teórico y metodológico que utilizan parte de una perspectiva macrosocial, que busca establecer nexos entre las estructuras económicas, políticas, sociales y el fenómeno educativo. Aunque el intento es válido, requiere de un mayor grado de profundidad, pasando del esquematismo a la ubicación de los entronques específicos entre la educación y las demás instancias de lo social.

El acento que los autores dan a lo macrosocial, lleva a que dejen de lado el manejo cuidadoso de la documentación educativa presentando solo realidades fragmentarias. Si bien esta obra tiene un gran valor dentro de un campo escasamente explorado, tan solo constituye un punto de partida cuyas ausencias deberán delimitarse.

El capítulo que dedican a los años en que transcurrió la República Liberal lo dividen en tres partes; la primera la constituye la administración de Olaya Herrera, que sin grandes modificaciones preparó el terreno de las reformas que se dieron en el siguiente intervalo durante la presidencia de Alfonso López Pumarejo -1934-1938_, esta segunda fase representó el momento más activo en el propósito de adecuar" el sistema educativo a la estructura productiva engendrada por la industrialización "69.

Su opinión de la reforma educativa del 36 es bastante optimista, retoma sólo los hechos que la destacan como positivista, esquivando los obstáculos e inconsistencias anotadas por otros investigadores. Los años correspondientes a los gobiernos de Eduardo Santos y Alfonso López sucedidos entre 1938 y 1945, son agrupados en una tercera etapa que representó un ritmo más lento, como lo demuestra su actuar en materia educativa

\footnotetext{
${ }^{66}$ Ibid., p. 289.

${ }^{67}$ GONZALEZ, Fernán. Educación y Estado en la historia de Colombia. Cinep, Bogotá, 1978. Serie Controversia, No. 77-78.

${ }^{68}$ MOLANO Y VERA. Evolución de la política educativa en el siglo XX. Universidad Pedagógica, Bogotá, 1982.

${ }^{69}$ lbid., p. 112.
} 
circunscrito al factor cuantitativo. Molano y Vera concluyen que "pese a sus fracasos e inconsistencias", la política educativa del período" revolucionó profundamente el sistema educativo", lo modernizó, democratizó y situó en los umbrales del siglo $\mathrm{XX}^{70}$.

Una de las investigaciones más brillantes sobre educación en las primeras décadas de este siglo es la de la historiadora Aline Helg $(1984)^{71}$, quien introdujo al caudal investigativo de la historia de la educación nuevos rumbos. Su tesis doctoral comprende casi cuarenta años -1918-1957-, la primera novedad consiste en emplear una periodización diferente a las administraciones liberal-conservadora, al definir peculiaridades inherentes al proceso de la educación, que no coinciden necesariamente con el cambio de un gobierno a otro.

Su clasificación comprende cuatro fases correspondientes a direcciones diferentes en la política educativa: entre 1918 y 1924 se inicia una etapa en la que progresivamente se pone en acción la legislación de principios de siglo; el segundo sub-período va de 1924 a 1934 y se caracteriza por la iniciación de los procesos de reforma; el tercer momento lo ubica entre 1934 y 1938 con López Pumarejo y su programa de Revolución en Marcha, valorando "su tentativa de integración nacional a través de la educación" el último lapso lo sitúa entre 1938 y 1957 donde sucedieron una serie de transformaciones demográficas y socio-económicas que influyeron decisivamente en la educación.

Colocando como eje temático la educación primaria y secundaria pública y privada, Helg ordenó de manera rigurosa las principales disposiciones educativas, organizó las estadísticas más importantes, consultó y reseñó la mayoría de las fuentes de primera mano incluyendo las orales. La investigadora trabaja filones que hacía muchos años no eran excavados o lo habían sido de manera superficial; ahora ella tiene la posibilidad de extraer nuevos datos, reordenar y plantearse los problemas desde ángulos que están por encima de los sesgos de tipo partidista.

Para Helg la reforma educativa de López Pumarejo expresó coherencia con el plan general de la Revolución en Marcha, que pretendía "desarrollar y modernizar al país para volverlo competitivo con el mercado capitalista mundial sin tocar las bases de la estructura social"72. Pero las materializaciones de la reforma tuvieron grandes limitaciones y si observamos el título que da a su obra en francés: "Civiliser le peuple et former les élites", podemos subrayar su modo de pensar sobre la inclinación de la política educativa en la primera mitad del siglo; con este postulado alude a la progresiva cristalización de dos redes educativas, cuyos fines y logros son diferentes. Idea que participa de los principios de la teoría de la reproducción de Pierre Bourdieu ${ }^{73}$, quien se refiere a la existencia en las sociedades capitalistas de dos canales de formación, uno por el que pasarían los cuadros de las clases dominantes y otro que recorrerían los sectores populares, senderos en los que el grado de profundización en los conocimientos y adquisición de habilidades difieren ostensiblemente. Mirada que rompe el encanto de una educación que abre puertas a la escalada social, al revelar los condicionamientos económicos y sociales que la determinan ${ }^{74}$.

\footnotetext{
${ }^{70}$ Ibid., p. 114.

${ }^{71}$ HELG, Aline. Civiliser le peuple et former les élites (1918-1957). Editions L'Harmattan, Paris, 1984. Actualmente el CEREC adelante su traducción al español.

La educación primaria y secundaria durante el primer gobierno de Alfonso López Pumarejo (1934-1938)". En: Rey. Colombiana de Educación. (Bogotá), (6): 9-36, 1980.

${ }^{72}$ Civiliser... op. cit., p. 13

${ }^{73}$ BOURDIEU, P. y PASSERON, J.C. La Reproducción. Siglo XX, México, 1979.

74 "Educación de las clases populares.., pero en función del Estado, y no para poner en las manos del pueblo un instrumento absoluto de promoción y emancipación". MORA, Christiane. "La difusión de la cultura en la juventud de las clases populares en Francia
} 
El ensayo sociohistórico de Antonio García "La crisis de la Universidad" (1985) ${ }^{75}$, expone desde un enfoque estructuralista seis modelos por los que ha pasado la universidad colombiana y asigna a la República Liberal el funcionamiento de un modelo universitario de corte liberal enmarcado en los complejos procesos de modernización capitalista que sacudían a Colombia. Aunque en escasas páginas delinea el perfil de la República Liberal y sus especificidades en educación, maneja una visión global del problema e incluye cifras sobre algunos niveles del sector, extractadas del informe de la misión Currie y de datos estadísticos de la Contraloría. Ante un tema tan poco indagado, esta obra es una valiosa referencia que sugiere caminos a quienes deseen continuar la búsqueda investigativa.

No es posible dejar de mencionar la acepción que sobre el período maneja Luis A. Bohórquez (1956) ${ }^{76}$, pedagogo conservador para quien el año de 1930 representó un cambio "fundamental» en materia educativa, signado por la Instauración de "monopolios estatales en la educación", afirmación bastante ambiciosa si consideramos el grado de debilidad del Estado en el plano educativo para la época. Bohórquez señala en el tipo de formación impartida en las aulas escolares el surgimiento de una generación confusa e indisciplinada que trajo consecuencias funestas para la patria en abril de 1948. Al indicar lo que pudieran ser los rasgos positivos, asegura que quedaron anulados por las resistencias generadas producto de la inclinación que tenían, en la que predominaba el deseo por "alejar la moral cristiana de la educación"77.

En vastos sectores de la jerarquía eclesiástica hubo consenso sobre este asunto; hacia la misma dirección apuntaron, por ejemplo, los comentarios del ministro y parlamentario conservador Joaquín Estrada Monsalve: "puede afirmarse que el 9 de abril fue lentamente elaborado, desde 1936, en el despacho del ministro de educación. Desde allí se descompuso el alma de las generaciones que irrumpieron en la noche roja"78. En las pastorales eclesiásticas y en la Revista Interamericana de Educación se expresó también esta connotación sobre los hechos ${ }^{79}$.

Al hacer un balance parcial sobre los estudios realizados y sus aproximaciones al objeto de reflexión, podemos decir que la mayoría señala el período de la República Liberal como significativo para la educación en sus posibilidades de replanteamiento y cambio, incrustadas en un momento histórico en el que se tejió una intrincada red de transformaciones políticas, económicas, sociales e ideológicas.

No existe un acuerdo entre los estudiosos del tema sobre el alcance de las reformas educativas y de su naturaleza, algunos las consideran parciales y de carácter democrático bastante limitado, para otros constituyen una verdadera ruptura con la educación anterior, mientras hay quienes centran sus inquietudes en los resultados "negativos" que tuvieron en la juventud que se formó en este lapso. Lo anterior es expresión de que no existe

desde hace un siglo: la acción de la Liga de la Enseñanza”. En: BERGERON, L. Niveles de cultura y grupos sociales. Siglo XXI, México, 1977.

${ }^{75}$ GARCIA, Antonio. La crisis de la Universidad Plaza \& Janes, Bogotá, 1985.

${ }^{76}$ BOHORQUEZ, Luis A.op. cit.

${ }^{77}$ lbid., pp. 440-445.

${ }^{78}$ ESTRADA MONSALVE, Joaquín. Así fue la Revolución. Citado por DE ROUX, Rodolfo. Una Iglesia en estado de alerta. SCCS, Bogotá, 1983.

${ }^{79}$ En la Conferencia Episcopal de 1948 la jerarquía eclesiástica manifestaba desacuerdo con el nombramiento de Gerardo Molina como rector de la Universidad Nacional, y lo tildaba de "Jefe comunista" imputándole responsabilidad en el desvío de la educación que recibió nuestra más brillante juventud de ese centro, hasta llegar a encabezar la subversión del orden constitucional el 9 de Abril". Conferencias Episcopales de Colombia, Tomo 1: 1908-1953. Bogotá. 
conocimiento ingenuo, que no esté consciente o inconscientemente comprometido; así, frente a la concepción que parece creer que los hechos hablan por sí mismos, encontramos que ellos hablan de diversa manera de acuerdo al ángulo desde el cual se les interrogue y de la problematización que en torno a ellos construya el investigador.

Los estudios han subrayado los caracteres que dieron forma a la dinámica educativa entre los años 1930-1 946, dejando indicadas relaciones en diversas direcciones que deberán ser escudriñadas con mayor detenimiento. Veamos un poco los alcances de sus planteamientos y los ámbitos que ellos han iluminado, para entrever cómo es posible continuar el trabajo alrededor de esta yeta histórica.

En líneas generales, es claro que ninguno de los trabajos mencionados se ubica específicamente en la República Liberal, sitúan la reflexión a lo largo del siglo XX o incluso son obras de carácter más general; indudablemente este hecho ha restado la posibilidad de un tratamiento sistemático así como la escogencia cuidadosa de un corpus documental amplio que exceda el seguimiento de la legislación educativa.

La periodización empleada es por lo común concomitante con las divisiones que para el terreno de la historia política han propuesto algunos investigadores. La mayoría de las obras dedicaron un capítulo aparte a la educación en la República Liberal habiendo reseñado en uno previo la Hegemonía Conservadora y después de 1946 tipificaron los procesos educativos en la Reacción Conservadora y en el Frente Nacional.

Esta secuencia parte de la coincidencia mecánica entre la historia política y la historia de la educación, únicamente Aline Helg tiene una propuesta que rastrea las cadencias que considera propias del fenómeno educativo.

El tratamiento dado a estos 16 años es bastante desigual; por lo regular la atención se fija en la primera administración de López Pumarejo, y en muchos de los casos se le considera como la política educativa general de los liberales, por ello no hay mayor interés en los otros años, porque ellos o prepararon el terreno de 1934 o continuaron abonándolo. Aunque de hecho el cuatrenio 1934-1938 representa uno de los momentos más álgidos, es imperioso avanzar en la caracterización de la educación en los otros subperíodos encontrándoles una identidad propia, que permita volver sobre el primer postulado con mayores fundamentos y hacer una lectura mucho más cuidadosa.

Las investigaciones se acercan a la problemática desde una perspectiva general y si exceptuamos la búsqueda de Aline Helg —referida a educación primaria y secundaria- y de Antonio García - sobre educación universitaria-, en ninguna de las otras se profundiza un eje temático particular.

En gran medida, se ha venido trabajando sobre un terreno aún desconocido, se gira en torno a las mismas formulaciones, sin que el conocimiento de los hechos históricos y su conceptualización tenga avances importantes. En ello influye el relativo desinterés que ha tenido la historia de la educación para las actuales generaciones de intelectuales, y también la preferencia a escoger como corpus documental fuentes de índole secundaria sin que éstas hayan hecho aportes apreciables en el trabajo sobre la información primaria, persistiendo la reflexión en el plano de la generalidad.

Paradójicamente este es un período que cuenta con abundante documentación, pues sin duda alguna, en la primera mitad del siglo la discusión sobre el problema educativo adquirió una dimensión nacional y se plasmó en un sinnúmero de publicaciones, cuyo 
estudio permitiría ir avanzando en un juego de luz y sombra, hacia las formas que plasmaron los entrelazamientos de la educación y otras esferas sociales. Es necesario conocer los distintos grupos que allí se expresaron, hay que ir tras las huellas que permitan reconstruir movimientos, tendencias, posiciones, personajes, que tomaron parte en el replanteamiento de la educación y de la cultura en una acepción mucho más amplía.

Las hipótesis están en buena medida en el nivel de formulación sugiriendo posibilidades para la comprensión histórica, pero requieren ser avaladas por otros estudios que permitan someterlas a amplia confrontación. Unas aluden a cuestiones como las relaciones entre educación e industrialización que deberán encontrar articulaciones más precisas que el simple reconocimiento de la incidencia del proceso de industrialización en curso sobre lo educativo. Hay que precisar los grados de esta readecuación, los requerimientos de la economía, el tipo de armazón del sistema educativo y los intereses en pugna.

Respecto a la participación creciente del Estado, se requiere delimitar su campo de acción, sus pretensiones y logros, la orientación educativa que desde él se difundía y las resistencias que se le oponían. Pues a pesar de la afirmación por todos conocida, de la función cada vez más activa que en este período juega el Estado, su nivel de intervención no fue uniforme y aún estamos lejos de determinar su real influencia en muchos de los espacios sociales.

Habría que puntualizar las afirmaciones sobre el elemento que trató de privilegiar la política educativa y si dirigió sus esfuerzos a los niveles de educación secundaria y superior o si enfatizó en primaria y alfabetización.

La legislación es sólo un indicador de fuerzas en tensión de las que hay que dar cuenta desde coordenadas que se alejen de aquella, con un horizonte más amplio que nos permita recuperar una gran riqueza documental para visualizar el panorama con un acervo de interrogantes multiplicado.

La educación primaria ha encontrado interesantes aportes en la obra de Aline Helg, al igual que lo que denominó formación profesional -que incluye enseñanza comercial, industrial y agrícola a nivel no universitario-. Además su esfuerzo sobre educación secundaria oficial y privada no tiene antecedentes.

En la actualidad no hay prácticamente trabajos sobre educación universitaria, que planteen fuera de la reestructuración de ja Universidad Nacional en 1935, la polémica de fondo sobre la introducción de nuevas corrientes de pensamiento, la dirección pragmática o humanista en la formación profesional, y el acceso a ésta de nuevos grupos sociales. Se necesita el análisis del proceso paulatino de profesionalización de ciertos oficios, como del papel jugado por las universidades privadas con orientación clerical. El libro de García es obligado punto de referencia, lo mismo que algunas menciones contenidas en la obra sociológica de Germán Rama, El sistema universitario en Colombia $(1970)^{80}$.

No existe un estudio exhaustivo de la formación de docentes, aspecto que fue relevante en la época, pues en ella se normatizaron asuntos pertinentes a la profesión del magisterio, al realce de su imagen social y de su función frente a los procesos de cambio; se crearon las escuelas normales rurales y se construyeron y readecuaron las normales regulares - urbanas-. Se fundaron tres facultades de educación con carácter universitario para preparar profesores de enseñanza secundaria y para el desempeño de

\footnotetext{
${ }^{80}$ RAMA, Germán. El sistema universitario en Colombia. Universidad Nacional, Bogotá, 1970.
} 
cargos directivos en el aparato educativo, éstas se fusionaron en la Escuela Normal Superior -1936-1951, entidad decisiva en la formación de una élite profesoral y en la institucionalización de las Ciencias Sociales en el país ${ }^{81}$. Los frutos de la Normal Superior son casi desconocidos por la producción historiográfica que en el mejor de los casos no le ha dedicado más de un párrafo ${ }^{82}$.

E' tema sobre ja educación femenina de notables progresos en el período, aún espera ser abordado. El movimiento estudiantil que tuvo un lugar protagónico en el cuestionamiento de los caducos pilares educativos y en la presión por obtener modificaciones de índole legislativa, tampoco ha llamado la atención de los investigadores.

Sobre el Ministerio de Educación habría que indicar el desarrollo de su constitución y reorganización, así como los engranajes a través de los cuales procuró centralizar y racionalizar el cuerpo educativo.

De gran utilidad serán los trabajos monográficos que ahonden sobre la educación en las diferentes regiones del país, ya que la información centralizada que progresivamente empezó a manejar el ministerio, ahogó las dinámicas desiguales en los distintos departamentos y territorios nacionales; si no se avanza en este sentido, cualquier intento de síntesis o balance general pisará terreno frágil.

Son pocas las relaciones comparativas con la educación en otros países, lo que refleja el manejo de un bagaje informativo de tipo localista, que no ha buscado respuesta a sus inquietudes desde derroteros más lejanos.

Los fenómenos ideológicos han sido tocados de manera fugaz, aún hace falta aclarar los basamentos doctrinales y filosóficos que permearon las ideas educativas del momento, pues la influencia del movimiento pedagógico de la Escuela Nueva tuvo sus formas particulares de apropiación por parte de los pensadores colombianos. Hay que comprobar además su grado real de influencia en los sujetos educativos y la práctica misma de estos ideales pedagógicos.

\section{III}

Para finalizar este panorama haremos algunas sugerencias a los estudiosos de la Historia de la Educación en Colombia interesados en el período de la República Liberal. Estas observaciones buscan llamar la atención sobre los requerimientos que hoy día posee el manejo de la temática, propendiendo porque se avance en la identificación y solución de los problemas que frenan el desarrollo de la producción histórica en este campo.

Los archivos sobre la historia de la educación deben ser explorados con rigor, enriqueciendo el acervo documental con que cuenta la comunidad de investigadores.

\footnotetext{
81 “Allí se formaron sistemáticamente, por primera vez en el país lingüistas, antropólogos, historiadores y otros científicos sociales... que, durante los cincuenta y los sesentas, contribuirían, en forma substancial a la consolidación de la Ciencia Social en Colombia". MELO, Jorge Orlando. "La historia de la ciencia en Colombia". En: Rey, de la Universidad de Antioquia. (Medellín), (203): 4-19 En.-Mzo., 1986.

${ }^{82}$ Para consultar una bibliografía detallada sobre la Normal Superior ver: HERRERA, Martha; LOW, Carlos y SUAR EZ, Hernán. "La Escuela Normal Superior: formar docentes con buen criterio. Entrevista a José Francisco Socarrás". En: Rey. Educación y Cultura. (Bogotá), (7): 20-26 Abril, 1986.
} 
Es necesario hacer lecturas menos sujetas al hilo de la legislación que amplien el espectro y posibiliten la incursión en múltiples terrenos.

Los estudios totales deben ser avalados por investigaciones que profundicen en el plano monográfico los diversos filamentos que integran la complejidad del haz educativo.

Se debe ahondar en los factores regionales y determinar las características de la educación en las distintas partes del territorio colombiano.

Es preciso avanzar en el inventario de los modelos culturales que buscó transmitir la escuela como forjadora de "sentido común".

Hay que estimular el conocimiento de los procesos educativos en otros países de América Latina y del mundo, que permita un acercamiento a la situación nacional desde una perspectiva comparativa.

Por último señalamos la pertinencia de sondear las duraciones propias del fenómeno educativo en su acaecer histórico, reformulando las posibilidades de periodización no sujetas mecánicamente a las de la historia política. 